\title{
Leakage current in high-purity germanium detectors with amorphous semiconductor contacts
}

\author{
Q. Looker, ${ }^{\mathrm{a}, \mathrm{b}, *}, \mathrm{M}$. Amman $^{\mathrm{a}}$, K. Vetter ${ }^{\mathrm{a}, \mathrm{b}}$ \\ ${ }^{a}$ Ernest Orlando Lawrence Berkeley National Laboratory, University of California, Berkeley, California \\ 94720, USA \\ ${ }^{b}$ Department of Nuclear Engineering, University of California, Berkeley, California 94720, USA
}

\begin{abstract}
Amorphous semiconductor electrical contacts on high-purity Ge radiation detectors have become a valuable technology because they are simple to fabricate, result in thin dead layers, block both electron and hole injection, and can readily be finely segmented as needed for applications requiring imaging or particle tracking. Though significant numbers of detectors have been successfully produced for a variety of applications using the amorphous semiconductor contact technology, there remains a need to better understand the dependence of performance characteristics, particularly leakage current, on the fabrication process parameters so that the performance can be better optimized. To this end, we have performed a systematic study of leakage current on RF-sputter-deposited amorphous-Ge (a-Ge) and amorphous-Si (a-Si) contacts as a function of process and operational parameters including sputter gas pressure and composition, number of detector temperature cycles, and time spent at room temperature. The study focused primarily on the current resulting from electron injection at the contact. Significant findings from the study include that a-Si produces lower electron injection than a-Ge, the time the detector spends at room temperature rather than the number of temperature cycles experienced by the detector is the primary factor associated with leakage current change when the detector is warmed, and the time stability of the aGe contact depends on the sputter gas pressure with a higher pressure producing more stable characteristics.
\end{abstract}

Keywords: Gamma-ray detectors, high-purity germanium, radiation detectors, semiconductor radiation detectors

* Corresponding author. Tel.: +1 5104952186.

E-mail address: qlooker@lbl.gov (Q. Looker). 


\section{Introduction}

For decades, radiation detectors based on high-purity Ge (HPGe) have been the preeminent technology for gamma-ray spectroscopy when excellent energy resolution and good efficiency are required [1][2][3][4]. Detectors of this type that are used solely for spectroscopy can be simple in design and typically consist of a single piece of HPGe onto which two electrical contacts have been fabricated. These contacts are used for bias voltage application and signal readout, and must block charge carrier injection so that low leakage current is achieved and associated electronic noise is reduced. Well established and reliable processes exist for manufacturing such contacts. The industry standard utilizes B implantation to form a $\mathrm{p}^{+}$, electron-blocking contact, while Li diffusion is used to form a thick and robust $\mathrm{n}^{+}$, hole-blocking contact. The success of this technology has enabled its use for a wide spectrum of tasks ranging from those of basic science to the highly applied activities found in industry.

More complex than the simple spectrometer detectors are those that, in addition to measuring energy, also determine the positions of the radiation interaction events in the detector. These position-sensitive HPGe detectors are needed for applications requiring imaging or particle tracking in addition to excellent spectroscopy. In such detectors, the position measurement is often achieved by dividing or segmenting the electrical contacts into many strips or pixels and then reading out the signals from each of the contact segments. The conventional B implanted contact can be readily segmented [5][6][7][8]. However, the Lidiffused contact presents a challenge for segmentation as a result of its thickness and significant diffusion of $\mathrm{Li}$ at room temperature [9][10]. Practically, the segmentation of the Li contact is limited to a granularity of no better than about $1 \mathrm{~mm}$ [7]. Modern applications are demanding increasingly fine position resolution, meaning smaller contact structures, thereby limiting the applicability of Li-diffused contacts. Although thin $\mathrm{n}^{+}$contacts have been developed using $\mathrm{P}$ implantation [11][12], they are difficult to produce and are less robust than either B-implanted or Li-diffused contacts.

The amorphous semiconductor electrical contact has emerged as an important technology capable of providing finely segmented contacts on HPGe detectors with both electron blocking and hole blocking properties, and is thereby capable of replacing both the B implanted contact and the problematic Li diffused contact. The technology allows a simple fabrication process where the HPGe crystal is first coated with a high resistivity thin film of an amorphous semiconductor such as amorphous $\mathrm{Ge}(\mathrm{a}-\mathrm{Ge}$ ) or amorphous $\mathrm{Si}$ (a$\mathrm{Si}$ ). This is then followed by depositing a patterned layer of metal, typically Al, on top of the amorphous film. The amorphous film dictates the charge blocking behavior of the contact and the metal defines the physical area of the contacts. Since the metal can be patterned using standard photolithographic processes, very fine contact segmentation can be achieved.

Amorphous Ge contacts were first experimentally investigated on crystalline Ge by Grigorovici et al. [13] and then later applied to Si radiation detectors by England et al. [14]. The use of the contacts on HPGe detectors was first demonstrated by Hansen and Haller in 1977 [15]. Hansen et al. also showed that a-Ge could be used to passivate the HPGe surfaces not covered with the electrical contacts and thereby control the charge state of these surfaces and make them less susceptible to change as a result of environmental exposure [16]. Over the years since the initial work on a-Ge, amorphous semiconductor contacts have been used to create position-sensitive detectors for x-ray and gamma-ray imaging [17][18][19][20][21], applied to Li-drifted Si detectors [22][23], shown to be appropriate for thin entrance window applications [17][24], used to create very fine contact segmentation [25], and enabled unique detector configurations such as field shaping [20] [21] and proximity electrode signal readout [26][27]. HPGe detectors with amorphous semiconductor contacts have been successfully used or are being developed for a wide variety of application areas including space science [28][29][30][31][32][33][34], material science [25], nuclear and particle physics [35][36][37] medical imaging [38][39], nuclear nonproliferation and homeland security [40][41][42][43], and environmental remediation [44].

Despite the success of the amorphous semiconductor contact, additional work is needed in order to better understand the relationship between the process parameters used when fabricating the contact and the 
performance characteristics of the detector. With this understanding, better optimized electrical contacts can be produced. Earlier work of this nature revealed that undesirable charge collection to the detector surfaces between segmented contacts on HPGe detectors could be reduced by adjusting the properties of the amorphous semiconductor layer [20] and that a-Si produces a better electron blocking contact than that obtained with a-Ge [45][46]. In the work presented in this paper, we have measured the bulk injected leakage current as a function of deposition parameters for RF-sputtered a-Ge and a-Si contacts. Our measurements have focused on the leakage current resultant from electron injection at negatively biased contacts. However, through a simple model of the contact, we argue that the results also provide useful information regarding the hole injection at positively biased contacts. Since our study focused on the impact of detector fabrication process parameters on detector performance, we first provide extensive details on the detector fabrication, detector testing, and data analysis. Following this, we present the results from our study and then end with a section summarizing the conclusions that can be drawn from our work.

\section{Experimental Methods}

\subsection{Detector Fabrication}

We fabricated a large set of small test detectors in order to examine the effect of altering amorphous film deposition parameters and identify critical factors determining performance and reliability. Multiple HPGe crystals from different sources were used, including material grown at LBNL several decades ago, ORTEC [47] material obtained more than a decade ago, and recently purchased ORTEC material. In this paper, detectors made from these materials will be referred to as LBNL1, ORTEC1, and ORTEC2, respectively. All of these materials were p-type with approximate impurity concentrations of $9 \times 10^{9}$, $1.8 \times 10^{10}$, and $7 \times 10^{9} \mathrm{~cm}^{-3}$, respectively. Several detector crystals were cut from a slice out of the three different boules. The main body of each detector crystal consisted of a square contact area measuring 18 $\mathrm{mm}$ on a side and a thickness perpendicular to the contact faces of about $10 \mathrm{~mm}$ (see Figure 1). In addition to this active volume of the detector crystal were thin extensions (handles) protruding from the bottom side of the crystal. The geometry of the crystal and contacts is such that, during operation as a detector, the depletion region within the crystal never extends significantly into the handles. Since the handles remain undepleted, surface damage to the handles will not introduce leakage current. Consequently, the handles simplify detector fabrication and mounting during testing by providing an area of the crystal that can be handled without negatively affecting the detector performance.

To convert a HPGe crystal into a test detector for our study, the crystal was first cut to the shape shown in Figure 1 using a diamond saw. Each of the exposed surfaces of the cut crystal was then lapped in order to remove any blade marks left by the cutting operation. The surface damage introduced by these mechanical processes was then removed by etching the crystal in a 4:1 nitric to hydrofluoric acid mixture. Following this surface polish etch, the crystal was again etched briefly in fresh 4:1 etchant, quenched in deionized water, rinsed in methanol, and blown dry with nitrogen in order to prepare the surfaces for the electrical contact depositions. During this etch and all subsequent processing steps, only the crystal handles were used to hold and manipulate the crystal. The crystal was then immediately loaded into an RF diode sputtering system. The first of two sputter depositions consisted of coating the top contact face and the sides of the crystal with a-Ge. The deposition was done with the crystal offset from the sputter target center and with rotation to ensure adequate coating of the crystal sides. The recipe used for this contact was approximately the same for all detectors and consisted of depositing the a-Ge with a 15 mTorr pressure of Ar with $7 \% \mathrm{H}_{2}$ gas mixture at a power of $300 \mathrm{~W}$. This produced a film thickness on the top face of about $300 \mathrm{~nm}$. The sputter target used was 8 inches in diameter and composed of $99.999 \%$ purity Ge obtained from American Elements [48]. After the top and sides were coated, the crystal was flipped over, and the bottom contact face was sputter coated with either a-Ge or a-Si. This bottom contact was the focus of our study, and the process used to deposit the film was varied from detector to detector. The sputter power was 
always $300 \mathrm{~W}$ for $\mathrm{Ge}$ and $400 \mathrm{~W}$ for $\mathrm{Si}$. The sputter gas was 0 to $35 \% \mathrm{H}_{2}$, with the balance being Ar, and the sputter gas pressure was varied from 7 to 23 mTorr. In order to minimize crystal heating during sputtering, the deposition consisted of a series of alternating 2-minute sputter deposition and 5-minute cool periods. After the detector crystal was completely coated with amorphous semiconductor, Al was thermally evaporated onto both the top and bottom surfaces of the crystal. Evaporation shadow masks were used to define a guard ring structure on the top face and a full-area coating on the bottom face as shown in Figure 1. The Al thickness used for both faces was approximately $50 \mathrm{~nm}$.

Each detector crystal was reprocessed and tested many times. This allowed us to test reproducibility when an identical process was used or to eliminate any possible crystal dependence when investigating the impact of specific process variations. Detector reprocessing consisted of removing the $\mathrm{Al}$, a-Ge, and a-Si layers with appropriate etchants and then following the fabrication sequence described above starting at the brief $4: 1 \mathrm{HNO}_{3}: \mathrm{HF}$ etch step.

\subsection{Measurement Procedure}

After each detector was fabricated, it was loaded onto a variable-temperature sample stage in a test cryostat (see Figure 2) and then cooled so that capacitance and leakage currents could be measured . The cryostat design is such that the detector and variable temperature stage are enclosed by an infrared shield held at a temperature near $77 \mathrm{~K}$. The temperature of the sample stage can be controlled from $79 \mathrm{~K}$ to greater than $200 \mathrm{~K}$. The measurement electronics used to characterize the detectors included Keithley picoammeters for current measurement on both the guard ring and center contact, and signal processing electronics for the readout of the signals from the center contact (see Figure 1). The signal readout electronics consisted of an AC-coupled charge-sensitive preamplifier followed by a commercial analog pulse-shaping amplifier. The signal readout allowed for the spectral characterization of the detector and the measurement of detector capacitance as a function of the applied detector voltage $\left(C-V_{a}\right.$ characteristic).

For each detector crystal, a $C-V_{a}$ characteristic was measured in order to determine the full depletion voltage and impurity concentration of the crystal. This characteristic was obtained by applying a constant voltage $V_{a}$ plus a small voltage step to the detector. In our measurements, we applied a negative voltage to the bottom contact so that depletion began at the top contact (for p-type HPGe). The voltage step riding on top of $V_{a}$ leads to a measured charge pulse that is proportional to the product of the voltage step amplitude and the capacitance between the center contact of the detector and the undepleted HPGe (or the bottom contact if the detector is fully depleted). Since the voltage step amplitude is known, the capacitance is determined by measuring the charge pulse amplitude. This measurement is repeated for a range of detector voltages to generate a $C$ - $V_{a}$ characteristic. As the magnitude of $V_{a}$ is increased from zero, the depletion region grows and the capacitance decreases. Once the depletion region reaches the bottom contact and the detector becomes fully depleted, the capacitance becomes constant with further increases in $V_{a}$. Assuming a constant impurity concentration throughout the crystal, the impurity concentration $N_{d}$ is then estimated from the extracted full depletion voltage $V_{f d}$ using the equation $N_{d}=2 \varepsilon V_{f d} / q t^{2}$, where $\varepsilon$ is the dielectric constant of Ge, $q$ is the magnitude of the electron charge, and $t$ is the detector thickness [2].

The leakage current in a fully-depleted HPGe detector arises from several mechanisms including hole injection at the positive electrical contact, electron injection at the negative contact, charge flow along the surface of the detector, and thermal generation in the HPGe. The primary aim of our study was to focus on the charge carrier injection behavior of one of the contacts on the detectors (typically electron injection at the bottom). The thermal generation of electron-hole pairs is reduced to a negligible level by cooling the detector, and a guard ring structure was used to separately measure the surface current component, leaving charge carrier injection at the contacts as the primary contributor to leakage current in the center contact. An example current density versus voltage $\left(J_{c^{-}} V_{a}\right)$ characteristic along with the corresponding capacitance (plotted as $1 / C^{2}$ as a function of $V_{a}$ ) measured from a detector are shown in Figure 3. At detector voltages below full depletion, there is no electric field at the bottom contact and hence no leakage current from 
injection at that contact. The leakage current in this case is predominantly from hole injection at the top center contact. However, once full depletion is reached (as indicated by the $1 / C^{2}-V_{a}$ characteristic) and an electric field appears at the bottom contact, a step in the current occurs. This step increase is solely the result of electron injection at the bottom contact. By measuring this electron injection current step as a function of fabrication process and operational parameters, we are able to identify and optimize parameters that affect this source of leakage current.

\subsection{Data analysis}

The parameter of direct interest in this study is detector leakage current, which can be plotted as a function of fabrication process or operational parameters. However, the leakage current is a strong function of detector temperature, which can vary with operational conditions. We use a thermionic emission model to describe the temperature and voltage dependence of the leakage current so that a couple of parameters can describe the leakage current injection of a contact over a range of temperatures and voltages. Much like a metal, charge conduction in the amorphous semiconductor of our electrical contacts predominantly takes place through electronic energy levels near the Fermi energy. This similarity in conduction then motivates the use of metal-semiconductor (MS) or Schottky theory [49] to describe the amorphous semiconductor contacts. A model of the amorphous-crystalline semiconductor (ACS) junction of this type was developed by Dohler and Brodsky [50][51][52] and then later successfully applied to the a-Ge contact on HPGe [53]. In this model, the current normalized by the contact area is given by

$$
J_{c}=J_{\infty}(T) \exp [-(\varphi-\Delta \varphi) / k T]\left[1-\exp \left(-q V_{a} / k T\right)\right]
$$

where $\Delta \varphi$ is the barrier lowering term added in this model:

$$
\Delta \varphi=\left[\left(2 q V_{b i}+2 q V_{a}+N_{d} / N_{f}\right) N_{d} / N_{f}\right]^{1 / 2}-N_{d} / N_{f}
$$

In the equations above, $T$ is the temperature, $\varphi$ is the energy barrier to charge carrier injection, $k$ is the Boltzmann constant, $N_{d}$ is the net ionized impurity concentration of the HPGe, $N_{f}$ is the density of localized energy states near the Fermi level in the amorphous semiconductor, $q$ is the magnitude of the electron charge, $V_{a}$ is the applied voltage, and $V_{b i}$ is the built in voltage at the contact.

Equation (1) is essentially the standard diode equation for a MS contact except for the pre-factor $J_{\infty}$ and the addition of the barrier lowering term $\Delta \varphi$. In the MS theory, $J_{\infty}(T)=A^{*} T^{2}$, where $A^{*}$ is the effective Richardson constant [49]. Since this term is dictated in part by the transition probabilities between energy states on each side of the contact, it is to be expected that the term will be different between the MS and ACS contacts. In the ACS model of Dohler and Brodsky [52], $J_{\infty}(T)$ was left as a parameter to be determined from measurements. In our data analysis, however, we need an explicit form for the temperature dependence and therefore retain the $T^{2}$ dependence predicted by the MS model. Consequently, we assume the following,

$$
J_{\infty}(T)=J_{o} T^{2}
$$

where the pre-factor $J_{o}$ is a constant to be determined from the measurements.

The barrier lowering term $\Delta \varphi$ accounts for the lowering of the energy barrier as a result of electric field penetration into the amorphous semiconductor. In the case of a metal, this penetration is negligible, and there is no barrier lowering; the $J_{c}-V_{a}$ curve is flat unless one accounts for the image charge. For our 
detectors, the barrier lowering due to electric field penetration in the amorphous layer is clearly evident in the $J_{c^{-}} V_{a}$ characteristics as the continual increase in current with increasing detector voltage (see Figure 3).

We simplify Equations (1) and (2) by assuming that $q V_{a}>>k T, V_{a} \gg V_{b i}$, and $q V_{a}>>N_{d} / N_{f}$. Applying these assumptions to Equation (2), we obtain the following for the barrier lowering term:

$$
\Delta \varphi=\left[2 q V_{a} N_{d} / N_{f}\right]^{1 / 2}
$$

The above equation was derived for the case where the crystalline semiconductor is partially depleted. The electric field magnitude at the contact in this case is

$$
E=\left[2 q V_{a} N_{d} / \varepsilon\right]^{1 / 2} \quad,
$$

where $\varepsilon$ is the dielectric constant of the crystalline semiconductor. Combining Equations (4) and (5), we obtain

$$
\Delta \varphi=\left(\varepsilon / N_{f}\right)^{1 / 2} E
$$

Equation (6) holds for the case of a partially or fully depleted detector, as long as the appropriate equation for $E$ is used. This is a direct consequence of the field continuity boundary condition at the contact that is applied during the solution of Poisson's equation when deriving $\Delta \varphi$ [51].

For the detector configuration shown in Figure 1, Equation (1) describes the leakage current density and Equation (4) describes the barrier lowering for hole injection at the top contact. In this case, the energy barrier would be that for hole injection, and we designate this as $\varphi_{h}$. We can describe the leakage current in the partially depleted detector by combining Equation (1) and Equation (4) to obtain

$$
J_{c}=J_{0} T^{2} \exp \left[-\left(\varphi_{h}-\left[2 q V_{a} N_{d} / N_{f}\right]^{1 / 2}\right) / k T\right]
$$

As shown in Figure 3, once full depletion is reached, there is a step in the current due to electron injection at the bottom contact in addition to the hole injection at the top contact. Equation (1) also describes the leakage current and Equation (6) is the expression for barrier lowering above full depletion, where $E$ is replaced by the appropriate equation for the electric field. In this case, the energy barrier would be that for electron injection, and we designate this as $\varphi_{e}$. Using Equation (1) with Equation (6) and the appropriate expression for $E$ for a fully depleted detector, we obtain the leakage current density from injection at the bottom contact:

$$
\Delta J_{c}=J_{0} T^{2} \exp \left[-\left(\varphi_{e}-\left(\varepsilon / N_{f}\right)^{\frac{1}{2}}\left(V_{a}-V_{f d}\right) / t\right) / k T\right]
$$

The overall leakage current above full depletion is then the sum of Equation (7) and Equation (8).

The goal of our data analysis was to determine $\varphi_{e}, J_{o}$, and $N_{f}$ for the bottom contact. First, $J_{c^{-}} V_{a}$ characteristics were measured at several different temperatures. The hole injection contribution to the current density was removed from each $J_{c^{-}} V_{a}$ curve by fitting the data below full depletion to Equation (7). Using the parameters extracted from the fit, the hole contribution to leakage current was estimated for all voltage values and subtracted from the $J_{c}-V_{a}$ data. At this point, only leakage current from electron injection remains and there should be no current below $V_{f d}$. With a fit to Equation (8), we obtained $N_{f}$ for 
the bottom contact, but not the separate values of $J_{o}$ and $\varphi_{e}$ since $J_{o}$ and $\varphi_{e}$ contribute to a single parameter in the fit. To obtain $J_{o}$ and $\varphi_{e}-\Delta \varphi$, a detector voltage is selected (typically the highest one used in the measurement), and the current density at this voltage as a function of temperature was fit to Equation (8). Finally, with $J_{o}$ and $\varphi_{e}-\Delta \varphi$ obtained from the $J_{c}(T)$ fit, $\varphi_{e}$ was calculated using the value of $N_{f}$ determined from the $J_{c}\left(V_{a}\right)$ fits.

One important implication of the contact theory described in this section is that the contact can block both electron and hole injection, and that the sum of the energy barrier heights for both these processes, $\varphi_{h}$ $+\varphi_{e}$, should be equal to the bandgap energy of crystalline Ge. This was shown to be true in a previous study of a-Ge and a-Si contacts on HPGe [45]. Consequently, extracting only the electron injection characteristics of the contacts as we are doing here in principle also provides the hole injection characteristics. For example, a contact that has a large barrier to electron injection and produces a small electron injection current when negatively biased will have a small hole barrier and greater hole injection when positively biased. Furthermore, a barrier height of approximately half the band gap energy will block equally well for electrons and holes and would be appropriate when it is desirable to produce a detector with only a single contact recipe for both negatively and positively biased contacts.

\section{Results}

\subsection{Electrical Contact Stability}

It was previously observed during the early production of detectors for instruments such as the Nuclear Compton Telescope [54][29][30][31] that the leakage current would increase each time the detector was warmed to room temperature and then cooled back down for testing. It was also observed that the magnitude of the change varied, depending on the length of time that the detector remained at room temperature and the fabrication recipe that was used to produce the amorphous semiconductor contacts on the detector. An example set of $J_{c^{-}} V_{a}$ characteristics exhibiting this behavior is shown in Figure 4. The detector used for these measurements had the standard a-Ge top contact sputtered at 15 mTorr and a bottom contact also of a-Ge sputtered at a pressure of 7 mTorr. To obtain the set of characteristics, the detector was taken through the cycle of cooling, $J_{c^{-}} V_{a}$ measurement, and warming to room temperature many times. The characteristics for the first ten cycles are shown in the figure. Each $J_{c}-V_{a}$ characteristic exhibits insignificant hole injection at the top contact and a clear step in current at about $900 \mathrm{~V}$ that is the result of electron injection at the bottom contact. Consequently, the increasing current step with each temperature cycle indicates a lack of stability for the bottom contact. For the first four cycles, the wait period at room temperature was approximately 28 hours. Each of these cycles produced nearly the same increase in the current. However, between cycles four and five there was a longer wait period at room temperature of about 244 hours. This led to a much greater increase in the current and indicates that the room temperature storage period plays a key role in the leakage current change.

The dependence of the leakage current on room temperature storage is further demonstrated in Figure 5 where we have plotted the normalized leakage current at a specific detector voltage as a function of the total time the detector was stored at room temperature. For example, the plot labeled with a sputter pressure of 7 mTorr (circle symbols) was obtained from the data of Figure 4 by choosing the current densities at $V_{a}$ $=2000 \mathrm{~V}$, dividing each of these by the value at the first temperature cycle, and then plotting these normalized currents as a function of the cumulative time the detector was stored at room temperature. The open symbols are for cycles when the detector was left under vacuum, and the filled symbols are for those cycles where the detector was exposed to atmospheric air while warm. From this plot it is evident that the storage time at room temperature rather than the temperature cycling dictates the leakage current change, and that the current appears to somewhat stabilize after several hundred hours of room temperature storage. Exposure to atmospheric air apparently has little to no effect on the current change. 
Also contained in Figure 5 are plots for detectors that are identical to the one just discussed except that the bottom contacts were sputtered at different pressures. It is clear that the sputter pressure plays a key role in the leakage current stability for a-Ge contacts, and that a higher pressure produces a substantially more stable contact. At 15 mTorr, a comparatively small increase was seen, while at 19 mTorr the direction of the change was reversed and the current decreased with time. The physical mechanism responsible for this sputter pressure dependence is currently unknown, though it is well known that the structural and compositional characteristics of sputtered a-Ge films vary with the sputter gas pressure [55][56][57]. For example, measurements of the film stress in RF magnetron sputtered a-Ge on polycarbonate substrates made by D. Fahnline et al. [55] demonstrated that the a-Ge films go from compressive to tensile stress as the Ar sputter pressure is increased from 5 to $25 \mathrm{mTorr}$, and that near stress-free films are obtained at about 12 mTorr. The compressively stressed films were also observed to have a greater Ar content and a smaller void fraction. Though the quantitative measurements from this study are not directly applicable to our detectors, the observation of changing film properties such as the stress changing from compressive at low sputter pressures to tensile at high pressures is commonly reported and is likely the case for our sputtered films. A future study combining detector measurements and amorphous film material analysis will be required in order to determine whether film stress or some other film property is the cause of the contact instability that we observe.

The results shown in Figure 5 were obtained using only ORTEC1 material. However, the dependence of the stability on sputter pressure was also confirmed through measurements with the other detector materials. For example, an ORTEC2 detector with the bottom contact sputtered at 7 mTorr exhibited a current that increased by a factor of about 7 after approximately 700 hours at room temperature. Moreover, a detector produced from the same material with the bottom contact sputtered at 15 mTorr was much more stable and showed a current that decreased by a factor of about 0.5 after approximately 450 hours at room temperature. Furthermore, an LBNL1 detector produced with contacts sputtered at 15 mTorr had stable current that changed by less than $6 \%$ after 170 hours of storage.

The addition of $\mathrm{H}_{2}$ to the Ar sputter gas can change the resistivity of the resultant a-Ge or a-Si layers by several orders of magnitude [45][58]. Since our detectors typically use the amorphous semiconductor layer as both the electrical contact and the HPGe surface coating between contacts, it is desirable for the layer to be high in resistivity so as to maintain good contact separation and low electronic noise. Consequently, the a-Ge and a-Si are sputtered in an Ar and $\mathrm{H}_{2}$ gas mixture. Since the contact stability could be affected by the concentration of $\mathrm{H}_{2}$, we measured the leakage current as a function of the cumulative time at room temperature for detectors produced with different concentrations of $\mathrm{H}_{2}$ in the sputter gas. A single HPGe crystal was used for this study and was fabricated four times with the bottom contact process varied between each of the fabrication runs. The results from the measurements made on these detectors are shown in Figure 6. These results demonstrate that we have good run-to-run reproducibility in the leakage current stability and that the $\mathrm{H}_{2}$ concentration has little impact on the stability over the range from 0 to 17.5 $\%$. Over this concentration range, the detectors behaved nearly the same in that the current increased slightly over time. A detector made with a $35 \% \mathrm{H}_{2}$ concentration (not plotted in the figure) also exhibited good stability but had a current that decreased slightly with time.

Finally, we also investigated the time stability of detectors produced with the standard a-Ge top contact and an a-Si bottom contact. This was a less extensive study and consisted of a-Si contacts sputtered only at a pressure of 7 mTorr. A typical set of $J_{c^{-}} V_{a}$ characteristics for such a detector is shown in Figure 7 . Note that these characteristics were measured at a temperature of $140 \mathrm{~K}$ rather than the measurements of Figures 4 through 6 , which were made at $110 \mathrm{~K}$. This is because the a-Si contact is a much better blocking contact for electron injection than a-Ge. At $110 \mathrm{~K}$, the leakage currents from the a-Ge/HPGe/a-Si detectors were at or below the $\sim 1 \mathrm{pA}$ accuracy of our current measurements. Consequently, these detectors were tested at higher temperatures. From Figure 7 we see that at this higher temperature, there is significant leakage current below the full depletion voltage of $900 \mathrm{~V}$. This is from hole injection at the a-Ge contact. Unlike the a-Ge/HPGe/a-Ge detectors previously discussed, the hole injection at the top contact is no longer negligible 
because of the higher temperature and is comparable to the electron injection at the bottom contact. Figure 7 then provides an indication of the leakage current stability for the hole injection of the a-Ge contact and the stability for the electron injection of the a-Si contact. Since the current below full depletion and the current step at full depletion decrease with time, we conclude that both the hole injection of the a-Ge contact and the electron injection of the a-Si contact decrease with storage at room temperature. This result was typical of the measurements made on other a-Ge/HPGe/a-Si detectors where the HPGe material and the $\mathrm{H}_{2}$ gas concentration used for the a-Si contact were varied.

\subsection{Electrical Contact Barrier Height and Pre-factor}

In order to obtain additional information on the amorphous semiconductor electrical contacts and their dependence on the fabrication process, we analyzed the leakage current data obtained with our detectors using the ACS model and the procedure described previously in the data analysis section. The analysis procedure required the measurement of $J_{c}-V_{a}$ characteristics at several temperatures. An example set of characteristics from an a-Ge/HPGe/a-Ge detector produced from ORTEC1 material is shown in Figure 8, and the electron energy barrier height and pre-factor extraction from this data is shown in Figure 9. The slope of the line fit to the data points in Figure 9 is equal to $\left(-\varphi_{e}+\Delta \varphi\right) / k$. To determine $\varphi_{e}, N_{f}$ is needed, which is obtained from a fit of Equation (8) to the $J_{c}-V_{a}$ characteristics. Such an analysis of the Figure 8 characteristics gives $N_{f}=2 \times 10^{17} \mathrm{eV}^{-1} \mathrm{~cm}^{-3}$. Using this value for $N_{f}$, we obtain $\Delta \varphi=0.007 \mathrm{eV}$ for the barrier lowering term at $V_{a}=2000 \mathrm{~V}$. The corrected barrier height to electron injection is then $\varphi_{e}=0.33 \mathrm{eV}$ using this barrier lowering term and the slope of the fitted line in Figure 9. The pre-factor $J_{o}$ in Equation (8) is obtained from the y axis intercept of the fitted line in Figure 9. For this example, we obtained $J_{o}=75 \mathrm{~A} \mathrm{~cm}$ ${ }^{2} \mathrm{~K}^{-2}$.

A large set of detectors each with either an a-Ge or an a-Si bottom contact were studied as just described. Overall, there was a small but statistically significant difference in the electron injection characteristics obtained for the two different amorphous semiconductor materials. The electron energy barrier averaged over a total of 34 detectors with a-Ge contacts was $0.30 \pm 0.03 \mathrm{eV}$, whereas the average energy barrier from a set of nine detectors with a-Si contacts was $0.34 \pm 0.01 \mathrm{eV}$. Furthermore, the average pre-factor for this same set of a-Ge detectors was $19 \pm 33 \mathrm{~A} \mathrm{~cm}^{-2} \mathrm{~K}^{-2}$, compared to an average of $0.026 \pm$ $0.045 \mathrm{~A} \mathrm{~cm}^{-2} \mathrm{~K}^{-2}$ for the a-Si pre-factor. The larger barrier and smaller pre-factor of the a-Si contact combine to produce a much lower electron injection current as compared to the a-Ge contact. This is also clear when comparing the full depletion current steps in Figures 4 and 7. The current step in Figure 7 that results from electron injection at the a-Si contact is smaller than that of the a-Ge contact of Figure 4 despite the $30 \mathrm{~K}$ higher temperature of the a-Si measurement.

As stated previously in the analysis section, it is expected based on the MS and ACS theories that the sum of the electron energy barrier height and the hole energy barrier height for a particular contact should equal the bandgap energy of crystalline Ge. Since the average electron barrier energy of the a-Ge contact is significantly less than half of the bandgap, we expect that the hole barrier for this contact will be large and that the contact will be good for blocking hole injection. The low leakage currents of Figures 4, 7, and 8 obtained at voltage biases below full depletion support this expectation. At these voltages, the leakage current is almost exclusively from hole injection at the top a-Ge contact. This leakage current is several orders of magnitude less than that of the electron injection at the bottom a-Ge contact for the detector of Figure 8. Furthermore, we have analyzed the below full depletion leakage current of the Figure 8 detector at temperatures of $130 \mathrm{~K}$ and above, and extracted an energy barrier height of $0.36 \mathrm{eV}$ for hole injection at the top a-Ge contact. This result that a-Ge produces a good hole blocking contact as well as the result that a-Si is superior for electron blocking are consistent with work from a previous study comparing the barrier heights of a-Ge and a-Si on HPGe [45].

A more detailed look at the electron blocking characteristics of the a-Ge and a-Si contacts is provided in Figures 10 through 13 where we plot the barrier heights and pre-factors as a function of the sputter gas $\mathrm{H}_{2}$ 
concentration (Figures 10 and 11) and as a function of sputter gas pressure (Figures 12 and 13). In these figures, the markers indicate data points, and the lines are drawn through the average values. It is clear that for a particular set of process parameters there is significant scatter in the data. Possible causes of this include run-to-run variations in the detector surface preparation and condition, errors in controlling the sputter conditions (for example, $\mathrm{H}$ from residual gas left in the chamber), and a dependence on the HPGe material. Despite this scatter, there are noticeable trends in the blocking characteristics. In Figures 10 and 11, data is shown only for either a-Ge contacts sputtered at 15 mTorr or a-Si contacts sputtered at 7 mTorr. At least two significant findings are apparent from the $\mathrm{H}_{2}$ concentration figures. First, for the a-Ge contacts, there is a drop in the barrier height as the concentration is increased from near zero to $17.5 \%$. This effect was also observed previously [45]. The barrier height reduction by itself could lead to a considerable increase in the electron injected leakage current. However, as the barrier height decreases there is also a corresponding reduction in the pre-factor. Depending on the detector temperature, these counteracting dependencies can lead to an electron injection current that has a minor dependence on sputter gas $\mathrm{H}_{2}$ concentration. At the higher $\mathrm{H}_{2}$ concentration of $35 \%$, the barrier height has returned to a higher value while the pre-factor has remained low. The combination of these two effects produces a lower leakage current contact. The second main finding from Figures 10 and 11 is that, in contrast to the a-Ge contacts sputtered at a pressure of $15 \mathrm{mTorr}$, the a-Si contacts sputtered at $7 \mathrm{mTorr}$ do not appear to be substantially affected by the $\mathrm{H}_{2}$ concentration of the sputter gas.

In Figures 12 and 13, data are shown for a-Ge and a-Si contacts sputtered at various pressures of Ar containing $7 \% \mathrm{H}_{2}$. For the a-Ge contacts, the barrier height does not appear to be substantially affected by the sputter gas pressure. The pre-factor however does show a discernible trend in that it decreases with increasing sputter pressure. For the a-Si contacts, the number of data points is small. This, combined with the scatter in the data, makes any conclusion regarding a trend with sputter gas pressure uncertain.

\section{Summary}

Driven primarily by the need for HPGe radiation detectors capable of good position resolution, the use of amorphous semiconductor electrical contacts on the detectors has grown substantially over the last decade. The technology has been successfully used to implement a wide range of detector configurations and the resultant detectors utilized in many different application areas. Despite this success, the performance of detectors made with amorphous semiconductor contacts could benefit from a better understanding of their operation and further optimization of their characteristics. The study presented in this paper has worked toward this goal by measuring the electron injection leakage current from RFsputtered a-Ge and a-Si contacts on HPGe as a function of fabrication process and operational parameters.

For many applications, a critical detector feature is the stability of the electrical characteristics (for example, leakage current) regardless of temperature cycling and extended storage at room temperature. Depending on the process used to produce the amorphous contacts, the leakage current through the contacts has been observed to change significantly when the detectors were cycled to temperature and re-tested. A key finding of our study is that it is the total time stored at room temperature rather than the temperature cycling that is the relevant parameter affecting the change in the charge injection at the contacts. We further observed that the current change with time typically diminished and appeared to approach a stable state. For electron injection from a-Ge contacts, the stability was strongly dependent on the sputter gas pressure during deposition of the a-Ge. A low pressure of $7 \mathrm{mTorr}$ gave poor stability in that the leakage current increased by about a factor of ten with extended room temperature storage, whereas higher pressures gave good stability. Hydrogen gas is typically added to the sputter gas in order to passivate defect states and thereby increase the resistivity of the amorphous layers. For the deposition conditions tested, the $\mathrm{H}_{2}$ concentration of the sputter gas did not appear to play a significant role in the electron injection stability of the a-Ge contact. For electron injection at the a-Si contacts, a sputter pressure of $7 \mathrm{mTorr}$ produced contacts 
that generally had a decreasing leakage current with time spent at room temperature. For the range of sputtering parameters used in this study, we found that the yield of functional detectors did not vary significantly with the choice of the sputter pressure or hydrogen content in the tested range.

Using a modified metal-semiconductor contact model, the electron injection leakage currents from a-Ge and a-Si contacts were analyzed and electron energy barrier heights and pre-factors were extracted. We determined that the electron barrier height is generally higher for a-Si than a-Ge while the pre-factor is generally lower for a-Si. The combination makes a-Si better suited for producing a low leakage current electron blocking contact. Since the electron barrier heights measured from a-Ge contacts were all well below half the bandgap energy of crystalline Ge, it is expected that the hole barrier height will be larger than that for electrons. Both the low leakage currents obtained with positively biased a-Ge contacts and the hole barrier height extracted from our standard a-Ge contact support this expectation and demonstrate that the a-Ge contact is well suited for use as a hole blocking contact.

We further analyzed the dependence of the electron injection characteristics on sputter gas $\mathrm{H}_{2}$ concentration and sputter gas pressure. For a-Ge sputtered at a pressure of 15 mTorr, both the electron barrier height and the prefactor decreased as the $\mathrm{H}_{2}$ concentration was increased from near zero to $17.5 \%$. For a-Ge sputtered with a $7 \% \mathrm{H}_{2}$ concentration, the electron barrier height was relatively constant with sputter pressure, whereas the pre-factor decreased with increasing pressure up to a pressure of about 15 mTorr. For the set of a-Si contacts studied, no pronounced dependence on $\mathrm{H}_{2}$ concentration or sputter pressure was observed.

\section{Acknowledgements}

We thank Paul Luke for useful discussions throughout this project. This work was supported by the U.S. Department of Energy, Office of Science, under contract number DE-AC02-05CH11231.

\section{References}

[1] K. Debertin and R. G. Helmer, Gamma- and X-Ray Spectrometry with Semiconductor Detectors, Amsterdam: Elsevier Science Publishers, 1988.

[2] G. F. Knoll, Radiation Detection and Measurement, New York: Wiley, 1989.

[3] G. Gilmore, Practical Gamma-Ray Spectroscopy, New York: Wiley, 2008.

[4] K. Vetter, "Recent Developments in the Fabrication and Operation of Germanium Detectors," Annual Reviews in Nuclear and Particle Science, vol. 57, pp. 363-404, 2007.

[5] P. N. Luke, "Gold-mask technique for fabricating segmented-electrode germanium detectors," IEEE Transactions on Nuclear Science, Vols. NS-31, no. 1, pp. 312-315, 1984.

[6] D. Protic and G. Riepe, "Position-sensitive germanium detectors," IEEE Transactions on Nuclear Science, Vols. NS-32, no. 1 , pp. 553-555, 1985.

[7] D. Gutknecht, "Photomask technique for fabricating high purity germanium strip detectors," Nuclear Instruments and Methods in Physics Research A, vol. 288, pp. 13-18, 1990.

[8] S. R. Amendolia, F. Bedeschi, E. Bertolucci and e. al, "Germanium microstrip detectors with 50 and 100 um pitch," Nuclear Instruments and Methods in Physics Research, vol. 226, pp. 117-121, 1984.

[9] J. T. Walton, R. H. Pehl, Y. K. Wong and C. P. Cork, "Si(Li) X-ray detectors with amorphous silicon passivation," IEEE Transactions on Nuclear Science, Vols. NS-31, no. 1, pp. 331-335, 1984.

[10] N. Q. Huy, D. Q. Binh and V. X. An, "Study on the increase of inactive germanium layer in a high-purity germanium detector after a long time operation applying MCNP code," Nuclear Instruments and Methods in Physics Research A, vol. 573, pp. 384-388, 2007.

[11] H. Herzer and S. Kalbitzer, "Ion Implanted High-Purity Germanium Detectors," Nuclear Instruments and Methods, vol. 101, pp. 31-37, 1972.

[12] R. H. Pehl and P. N. Luke, "High-purity germanium charged-particle detectors: A LBL-IUCF update," Nuclear Instruments and Methods in Physics Research A, vol. 242, pp. 103-110, 1985.

[13] R. Grigorovici, N. Croitoru, A. Devenyi and E. Teleman, Proc. 7th International Conference on the Physics of Semiconductors, p. 423, 1964.

[14] J. B. A. England and V. W. Hammer, Nuclear Instruments and Methods in Physics Research, vol. 96, p. 81, 1971. 
[15] W. L. Hansen and E. E. Haller, "Amorphous germanium as an electron or hole blocking contact on high-purity germanium detectors," IEEE Transactions on Nuclear Science, Vols. NS-24, no. 1, pp. 61-63, 1977.

[16] W. L. Hansen, E. E. Haller and G. S. Hubbard, "Protective surface coatings on semiconductor nuclear radiation detectors," IEEE Transactions on Nuclear Science, Vols. NS-27, no. 1, pp. 247-251, 1980.

[17] P. N. Luke and e. al., "Amorphous Ge Bipolar Blocking Contacts on Ge Detectors," IEEE Transactions on Nuclear Science, vol. 39, no. 4, pp. 590-594, 1992.

[18] P. N. Luke, R. H. Pehl and F. A. Dilmanian, IEEE Transactions on Nuclear Science, Vols. NS-41, p. 976, 1994.

[19] P. N. Luke, M. Amman, B. F. Phlips, W. N. Johnson and R. A. Kroeger, "Germanium orthogonal strip detectors with Amorphous-Semiconductor Contacts," IEEE Transactions on Nuclear Science, vol. 47, no. 4, pp. 1360-1363, 2000.

[20] M. a. L. P. N. Amman, "Position-sensitive germanium detectors for gamma-ray imaging and spectroscopy," Proceedings of the SPIE, 2000.

[21] M. Amman and P. N. Luke, "Three-dimensional position sensing and field shaping in orthogonal-strip germanium gammaray detectors," Nuclear Instruments and Methods in Physics Research A, vol. 452, pp. 155-166, 2000.

[22] I. D. Hau, C. Tindall and P. N. Luke, Nuclear Instruments and Methods in Physics Research A, vol. 505, p. 148, 2003.

[23] C. S. Tindall, M. Amman and P. N. Luke, IEEE Transactions on Nuclear Science, vol. 51, p. 1140, 2004.

[24] P. N. Luke, C. S. Rossington and M. F. Wesela, "Low Energy X-ray Response of Ge Detectors with Amorphous Ge

Entrance Contacts," IEEE Transactions on Nuclear Science, vol. 41, no. 4, p. 1074, 1994.

[25] J. Headspith and e. al., "First Experimental Data from XH, a Fine Pitch Germanium Microstrip Detector for Energy

Dispersive EXAFS (EDE)," IEEE Nuclear Science Symposium Conference Record, pp. 2421-2428, 2007.

[26] P. N. Luke, C. S. Tindall and M. Amman, "Proximity Charge Sensing With Semiconductor Detectors," IEEE Transactions on Nuclear Science, vol. 56, no. 3, pp. 808-812, 2009.

[27] M. Amman, A. Priest, P. N. Luke and e. al., IEEE Transactions on Nuclear Science, vol. 60, p. 1213, 2013.

[28] S. Boggs, M. Bandstra, J. Bowen and e. al., Exp. Astron., vol. 20, p. 387, 2005.

[29] W. Coburn, S. E. Boggs, J. D. Bowen and e. al., Proc. of SPIE, vol. 5898, pp. 589802-1, 2005.

[30] E. C. Bellm, S. E. Boggs, M. S. Bandstra and e. al., IEEE Transactions on Nuclear Science, vol. 56, p. 1250, 2009.

[31] E. C. Bellm, J.-L. Chiu, S. E. Boggs and e. al., Proc. of SPIE, vol. 7732, pp. 773224-1, 2010.

[32] M. S. Bandstra, E. C. Bellm, S. E. Boggs and e. al., Astrophys. Journal, vol. 2011, p. 8, 2011.

[33] A. Lowell, S. Boggs, A. Zoglauer and e. al., Proc. of SPIE, vol. 8443, pp. 84434U-1, 2012.

[34] A. Y. Shih and e. al., "The Gamma-Ray Imager/Polarimeter for Solar Flares (GRIPS)," Proceedings of SPIE, vol. 8443,

2012.

[35] N. Larson, S. N. Liddick, M. Bennett and e. al., Nucl. Instr. and Meth. A, vol. 727, p. 59, 2013.

[36] T. Shutt, J. Emes, E. E. Haller and e. al., Nucl. Instr. and Meth. A, vol. 444, p. 340, 2000.

[37] P. L. Brink, Z. Ahmed, D. S. Akerib and e. al., AIP Conf. Proc., vol. 1185, p. 655, 2009.

[38] L. C. Johnson and e. al., "Characterization of a high-purity germanium detector for small-animal SPECT," Physics in Medicine and Biology, vol. 56, pp. 5877-5888, 2011.

[39] F. A. Dilmanian, H. Rarback, E. Nachaliel and e. al., Conference Record of the 1992 IEEE Nuclear Science Symposium and Medical Imaging Conference, p. 1298, 1992.

[40] K. P. Ziock, N. Madden, E. Hull and e. al., IEEE Trans. Nucl. Sci., vol. 49, p. 1737, 2002.

[41] K. P. Ziock, M. T. Burks, C. P. Cork and e. al., Conference Record of the 2003 IEEE Nuclear Science Symposium, p. 1430, 2003.

[42] M. Cunningham, M. Burks, D. Chivers and e. al., Conference Record of the 2005 IEEE Nuclear Science Symposium, p. 312 , 2005.

[43] L. Mihailescu, K. M. Vetter, M. T. Burks and e. al., Nucl. Instr. and Meth. A, vol. 570, p. 89, 2007.

[44] B. F. Phlips, W. N. Johnson, R. A. Kroeger and e. al., IEEE Trans. Nucl. Sci., vol. 49, p. 597, 2002.

[45] M. Amman, P. N. Luke and S. E. Boggs, "Amorphous-semiconductor-contact germanium-based detectors for gamma-ray imaging and spectroscopy," Nuclear Instruments and Methods in Physics Research A, vol. 579, pp. 886-890, 2007.

[46] P. N. Luke, M. Amman, J. S. Lee and J. T. Walton, "Amorphous-Ge and amorphous-Si contacts on Ge detectors," IEEE Nuclear Science Symposium Presentation, 1998.

[47] "ORTEC Home Page," [Online]. Available: www.ortec-online.com.

[48] "American Elements," [Online]. Available: www.americanelements.com.

[49] S. M. Sze, Physics of Semiconductor Devices, New York: Wiley, 1981.

[50] M. H. Brodsky and G. H. Dohler, "A new type of junction: Amorphous/crystalline," Critical Reviews in Solid State and Materials Sciences, vol. 5, no. 4, pp. 591-595, 1975.

[51] M. H. Brodsky, G. H. Dohler and P. J. Steinhardt, "On the Measurement of the Conductivity Density of States of

Evaporated Amorphous Silicon Films," Phys. Stat. Sol., vol. 72, pp. 761-769, 1975.

[52] G. H. Dohler and M. H. Brodsky, Proc. International Conference Tetrahedrally Bonded Amorphous Semiconductors, p.

351, 1974.

[53] E. L. Hull and R. H. Pehl, "Amorphous germanium contacts on germanium detectors," Nuclear Instruments and Methods in Physics Research A, vol. 538, pp. 651-656, 2005. 
[54] S. Boggs and e. al., "Performance of the Nuclear Compton Telescope," Experimental Astronomy, vol. 20, pp. 387-394, 2005.

[55] D. Fahnline, B. Yang, K. Vedam, R. Messier and L. Pilione, "Instrinsic Stress in a-Germanium Films Deposited by RFMagnetron Sputtering," MRS Symposium Proceedings, vol. 130, pp. 355-360, 1989.

[56] R. Messier, T. Takamori and R. Roy, J. Vac. Sci. Technol., vol. 13, p. 1060, 1976.

[57] H. Windischmann, Critical Reviews in Solid State and Materials Sciences, vol. 17, no. 6, p. 547, 1992.

[58] W. Paul, A. J. Lewis, G. A. N. Connell and T. D. Moustakas, "Doping, Schottky barrier and p-n junction formation in amorphous germanium and silicon by rf sputtering," Solid State Communications, vol. 20, pp. 969-972, 1976.

[59] J. T. Walton and e. al., "Amorphous Silicon/Crystalline Silicon Heterojunctions for Nuclear Radiation Detector Applications," IEEE Transactions on Nuclear Science, vol. 44, no. 3, pp. 961-964, 1997. 


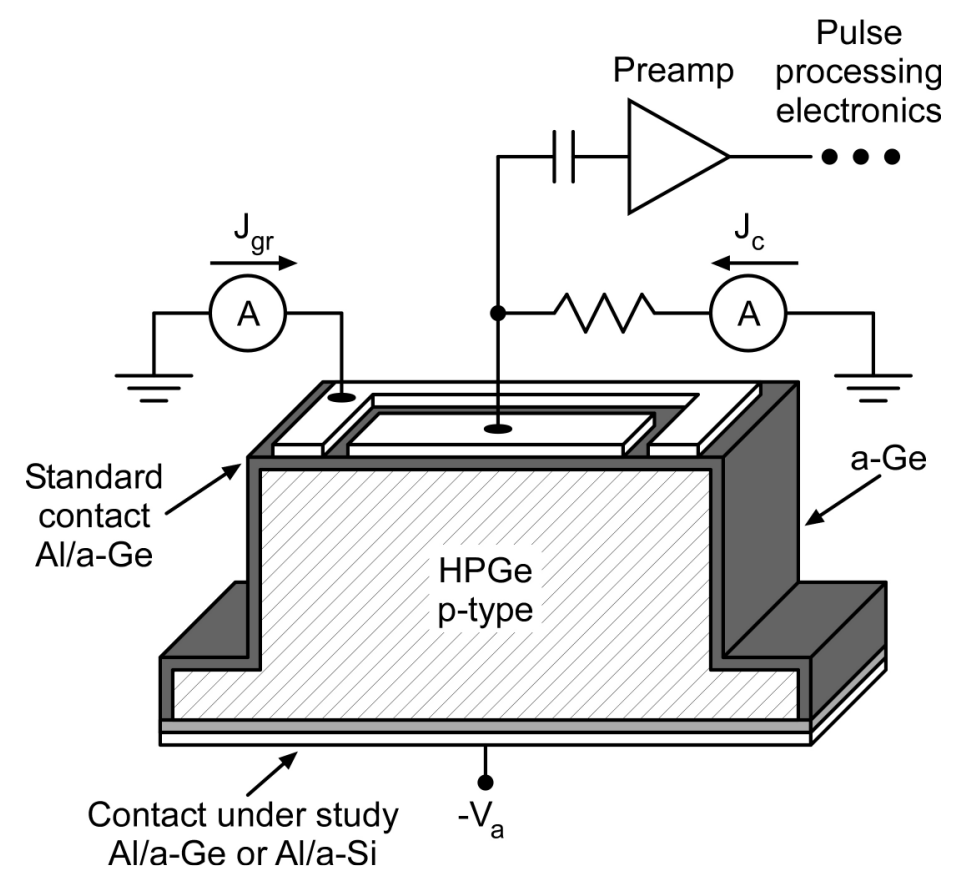

Figure 1. Schematic cross-sectional drawing showing the geometry of the HPGe detector and the measurement circuit. The top electrical contact was produced using the same process for all detectors whereas the bottom contact varied from detector to detector and was the contact being studied. The top contact had the Al layer patterned into separate center and guard ring electrodes. The Al layer on the bottom covered the entire contact surface.

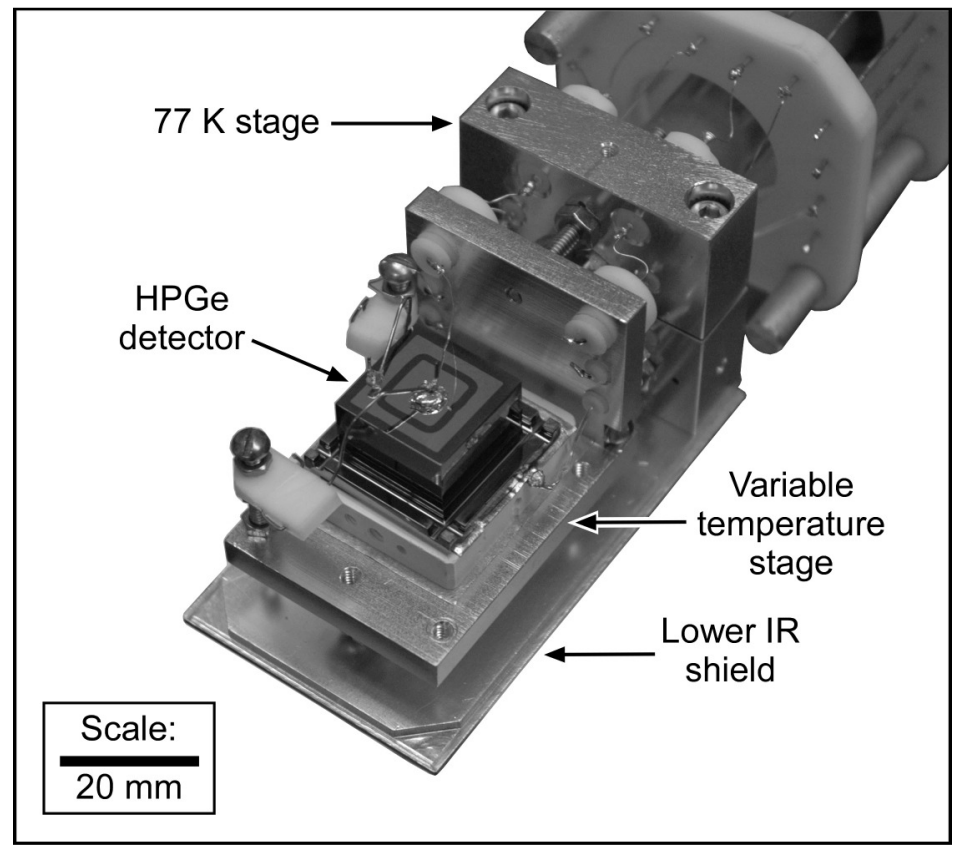

Figure 2. Photograph of a HPGe detector loaded into the test cryostat. The detector sits on a variable temperature stage that is surrounded by an infrared shield maintained at a temperature near $77 \mathrm{~K}$. 


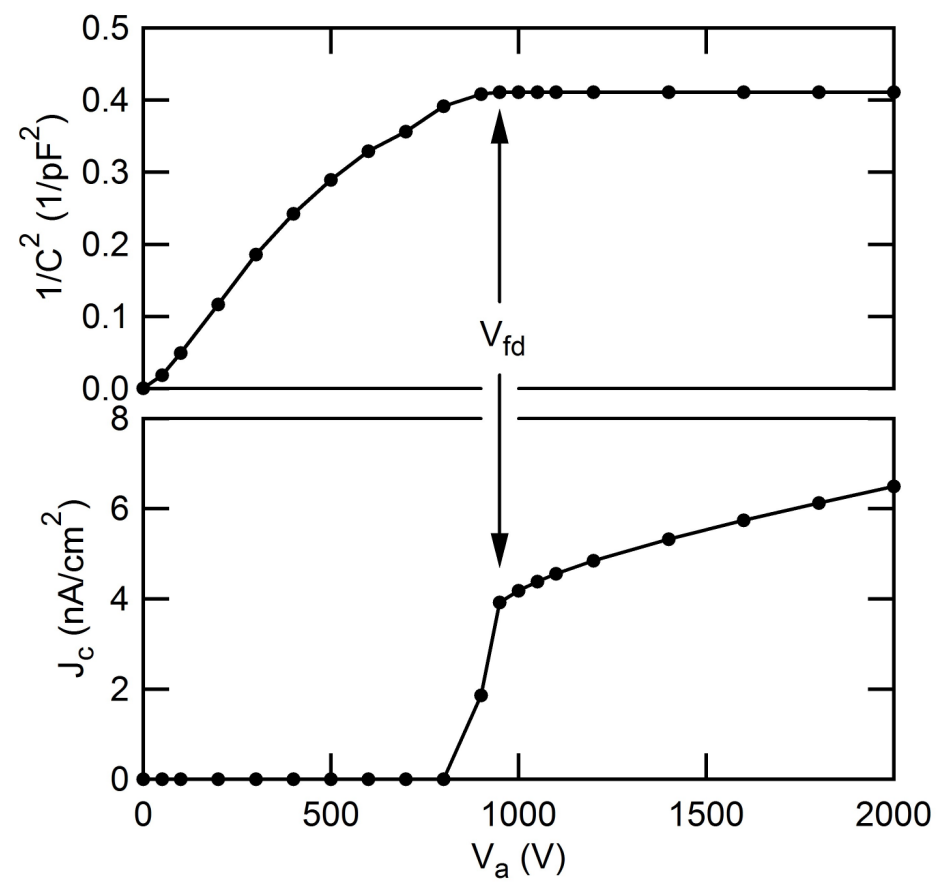

Figure 3. Measured center contact capacitance and leakage current density plotted as a function of bias voltage for a detector of the configuration shown in Figure 1. Both electrical contacts on the detector were a-Ge, and the HPGe crystal was ORTEC1 material. The measurements were made a temperature of $120 \mathrm{~K}$. 


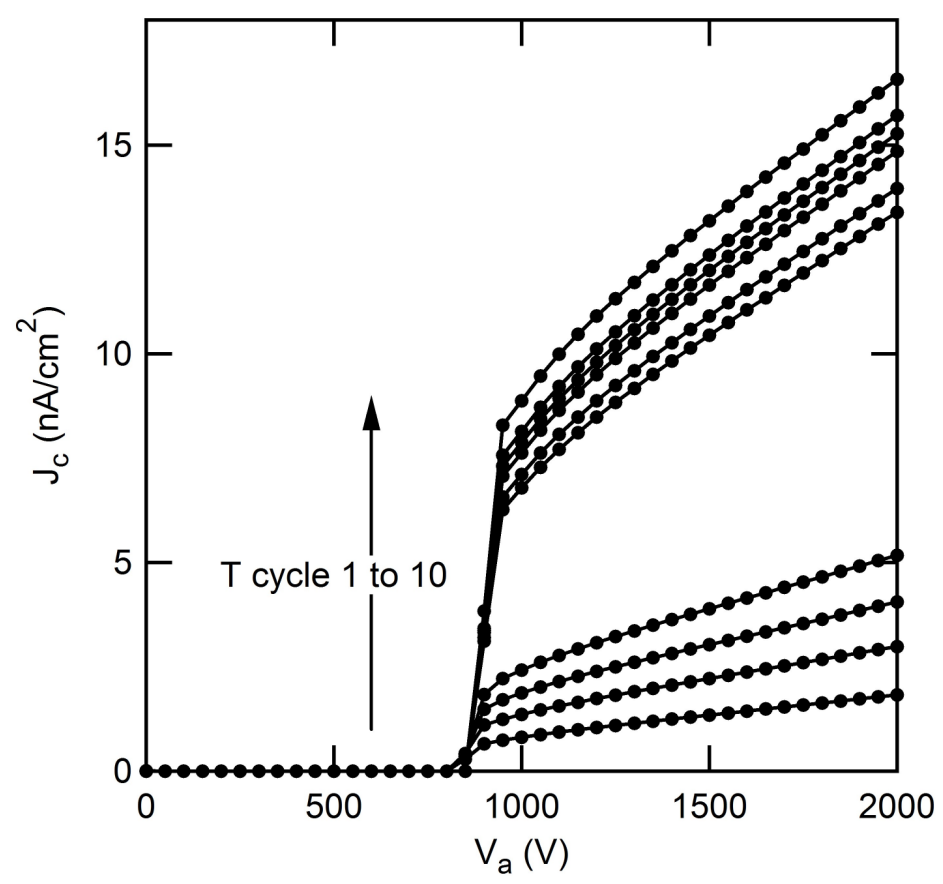

Figure 4. Measured center contact leakage current density plotted as a function of bias voltage for an a$\mathrm{Ge} / \mathrm{HPGe} / \mathrm{a}-\mathrm{Ge}$ detector of the configuration shown in Figure 1. The detector was temperature cycled many times, and the $J_{c}-V_{a}$ characteristic measured after each temperature cycle is shown. The measurements were made at a temperature of $110 \mathrm{~K}$. Both electrical contacts on the detector were a-Ge sputtered in Ar with 7 $\% \mathrm{H}_{2}$. The top contact was sputtered at a pressure of $15 \mathrm{mTorr}$ and the bottom at a pressure of $7 \mathrm{mTorr}$. The HPGe crystal was ORTEC1 material. 


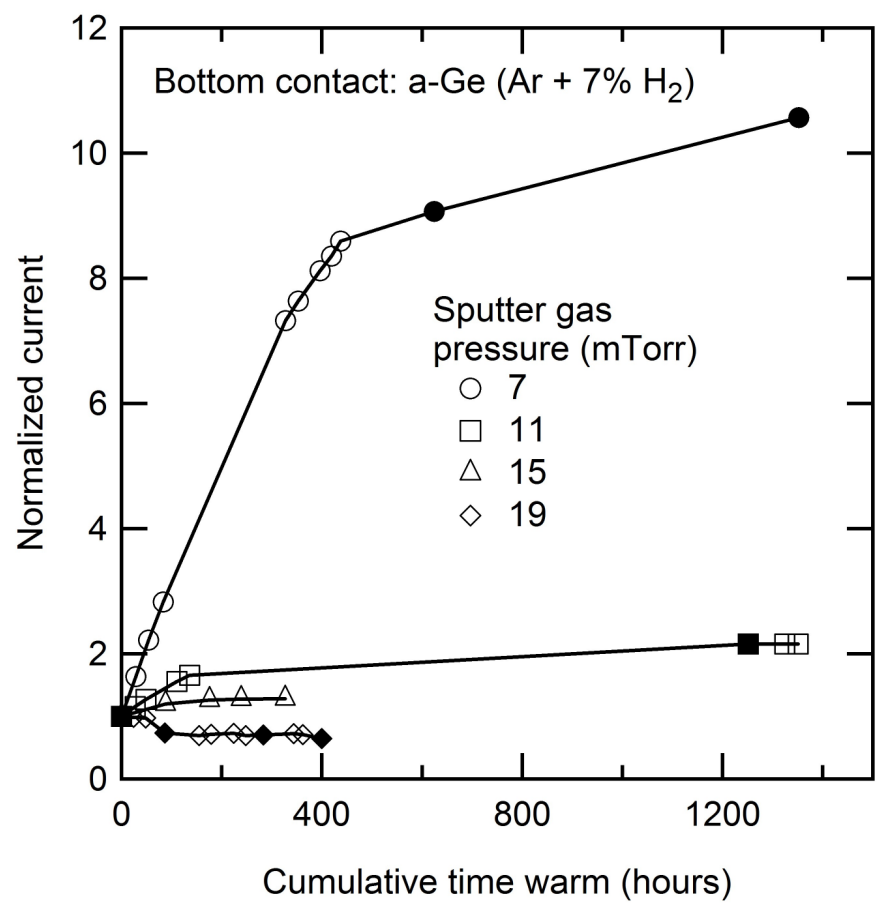

Figure 5. Measured center contact leakage current plotted as a function of time stored at room temperature for a-Ge/HPGe/a-Ge detectors of the configuration shown in Figure 1. The detectors were temperature cycled many times, and the current measured after each temperature cycle is shown. For each detector, the leakage current has been normalized to that measured during the first temperature cycle. Open symbols indicate that the detector was held under vacuum during the room temperature storage, whereas the closed symbols indicate that the detector was exposed to atmospheric air after the previous temperature cycle. Both electrical contacts on the detector were a-Ge sputtered in $\mathrm{Ar}$ with $7 \% \mathrm{H}_{2}$. The top contact was sputtered at a pressure of $15 \mathrm{mTorr}$ and the bottom at pressures of 7, 11, 15, and $19 \mathrm{mTorr}$ as indicated in the figure. The HPGe crystal was ORTEC1 material. The measurements were made at $V_{a}=2000 \mathrm{~V}$ and a temperature of $110 \mathrm{~K}$. 


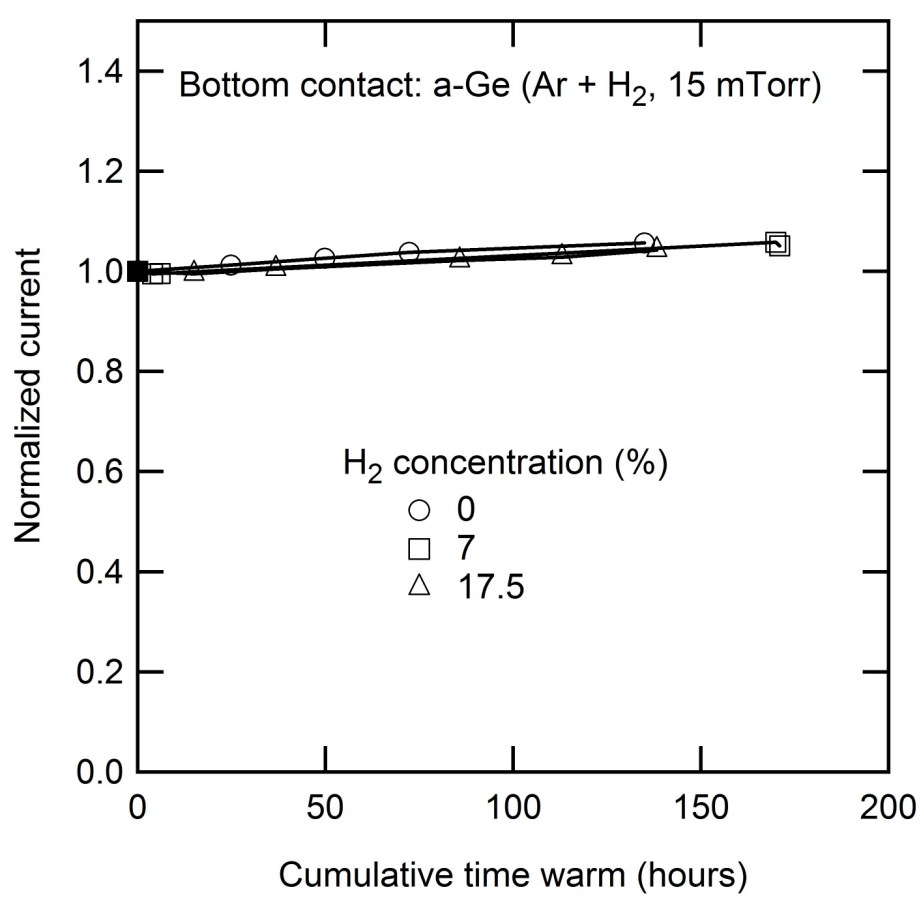

Figure 6. Measured center contact leakage current plotted as a function of time stored at room temperature for a-Ge/HPGe/a-Ge detectors of the configuration shown in Figure 1. The detectors were temperature cycled many times, and the current measured after each temperature cycle is shown. For each detector, the leakage current has been normalized to that measured during the first temperature cycle. Both electrical contacts on the detector were a-Ge sputtered at a pressure of $15 \mathrm{mTorr}$. The top contact was sputtered in Ar with $7 \% \mathrm{H}_{2}$ and the bottom at $\mathrm{H}_{2}$ concentrations of 0,7 , and $17.5 \%$ as indicated in the figure. The HPGe crystal was LBNL1 material. The measurements were made at $V_{a}=2000 \mathrm{~V}$ and a temperature of $110 \mathrm{~K}$. 


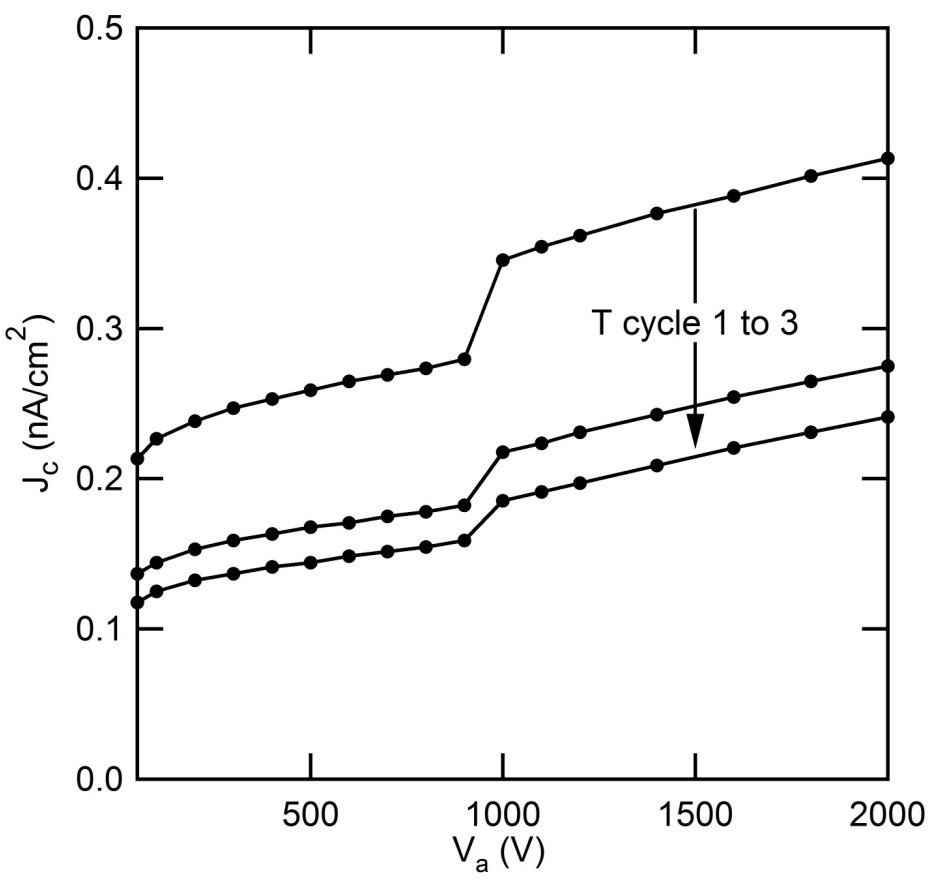

Figure 7. Measured center contact leakage current density plotted as a function of bias voltage for an a$\mathrm{Ge} / \mathrm{HPGe} / \mathrm{a}-\mathrm{Si}$ detector of the configuration shown in Figure 1. The detector was temperature cycled three times, and the $J_{c}-V_{a}$ characteristic measured after each temperature cycle is shown. The storage time at room temperature between the first and second cycle was 212 hours and between the second and third was 131 hours. The measurements were made at a temperature of $140 \mathrm{~K}$. The top electrical contact was a-Ge sputtered at a pressure of $15 \mathrm{mTorr}$, and the bottom contact was a-Si sputtered at a pressure of $7 \mathrm{mTorr}$. Both electrical contacts on the detector were sputtered in Ar with $7 \% \mathrm{H}_{2}$. The HPGe crystal was ORTEC1 material. 


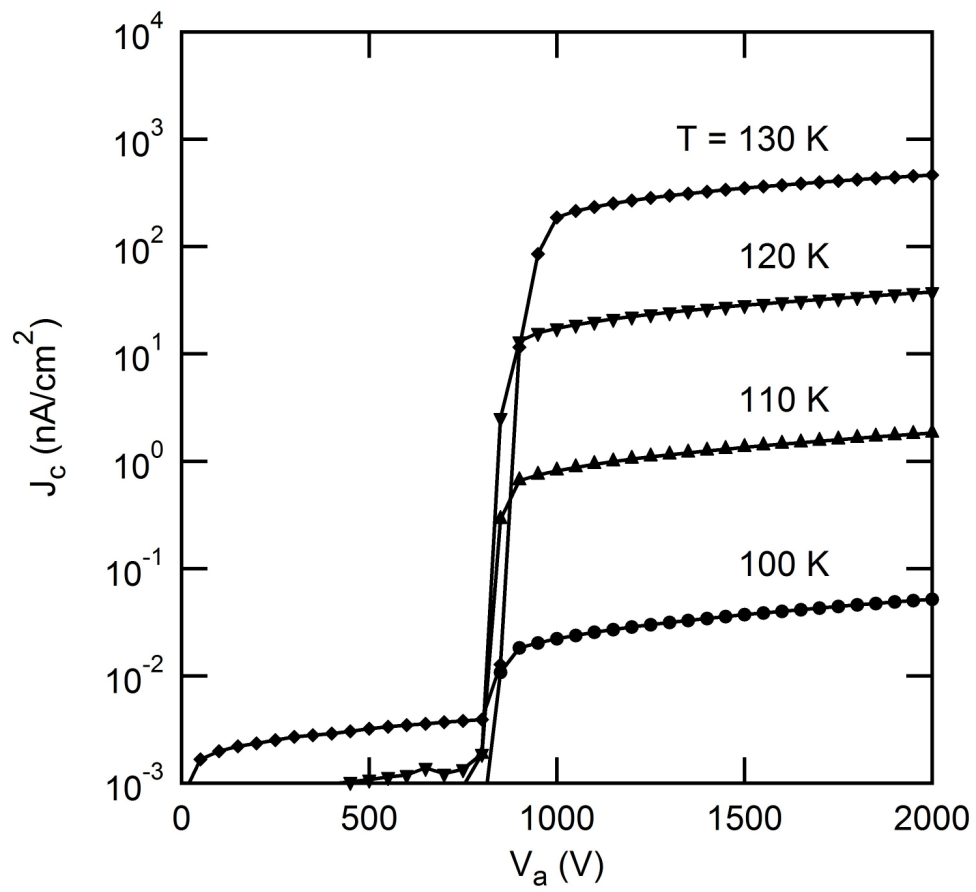

Figure 8. Measured center contact leakage current density plotted as a function of bias voltage at various temperatures for an a-Ge/HPGe/a-Ge detector of the configuration shown in Figure 1. Both electrical contacts on the detector were a-Ge sputtered in Ar with $7 \% \mathrm{H}_{2}$. The top contact was sputtered at a pressure of 15 mTorr and the bottom at a pressure of 7 mTorr. The HPGe crystal was ORTEC1 material. 


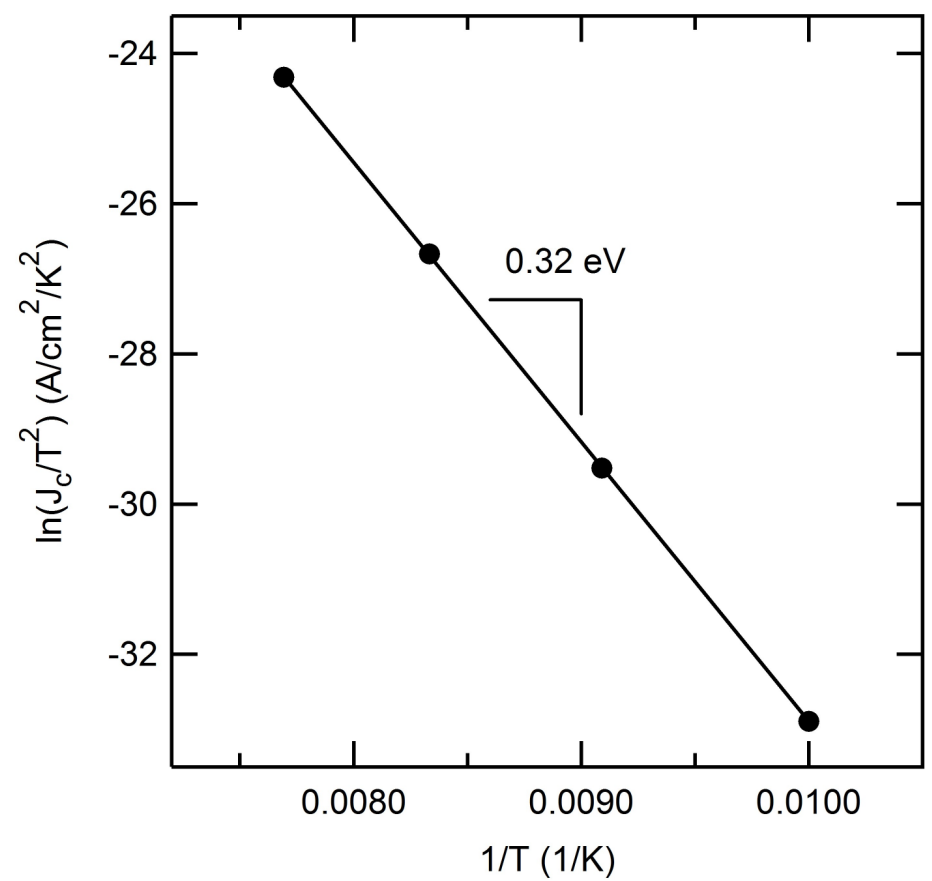

Figure 9. Plot illustrating the barrier height and pre-factor extraction from the data of Figure 8 using the method described in the data analysis section. The current density is that measured at $V_{a}=2000 \mathrm{~V}$. A linear fit to the plotted data gives a slope corresponding to the electron injection energy barrier. This barrier height must then be corrected by the barrier lowering term. The y axis intercept of the fitted line is the natural logarithm of the pre-factor $J_{o}$. 


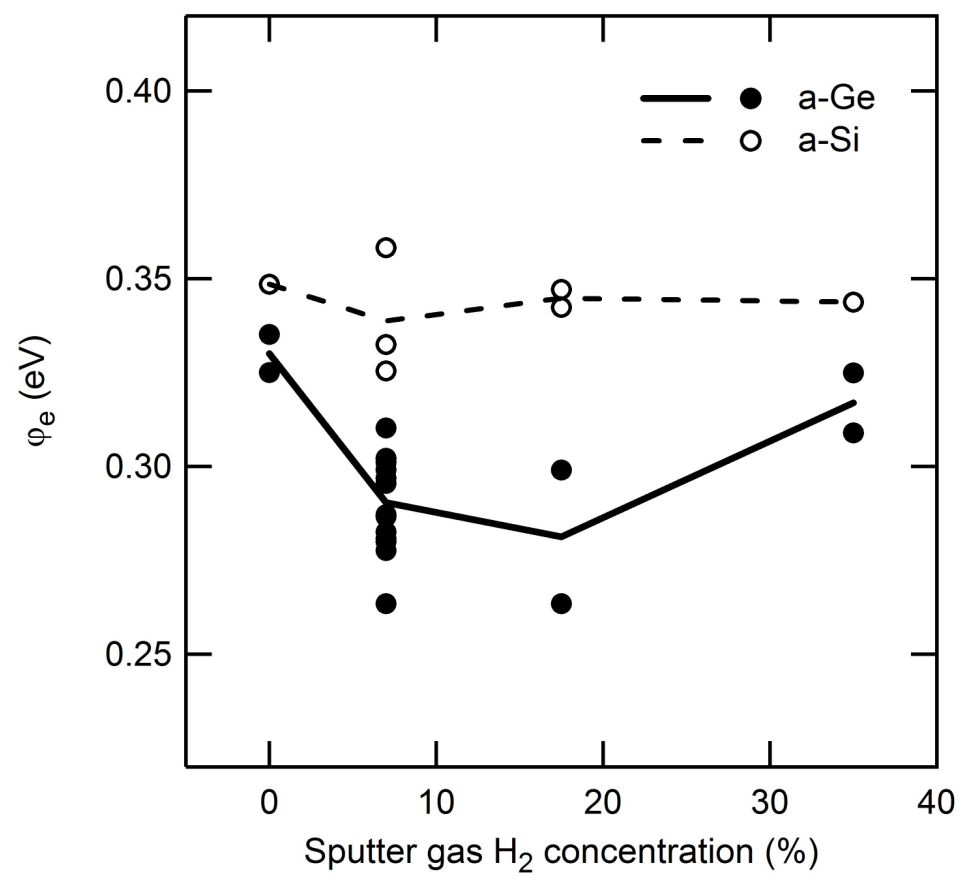

Figure 10. Electron energy barrier height plotted as a function of sputter gas hydrogen concentration for a$\mathrm{Ge} / \mathrm{HPGe} / \mathrm{a}-\mathrm{Ge}$ and a-Ge/HPGe/a-Si detectors of the configuration shown in Figure 1. The barrier height is that of the bottom contact. The a-Ge bottom contacts were sputtered at a pressure of $15 \mathrm{mTorr}$, and the a-Si bottom contacts were sputtered at a pressure of $7 \mathrm{mTorr}$. The data points include detectors produced from ORTEC1, ORTEC2, and LBNL1 HPGe materials. 


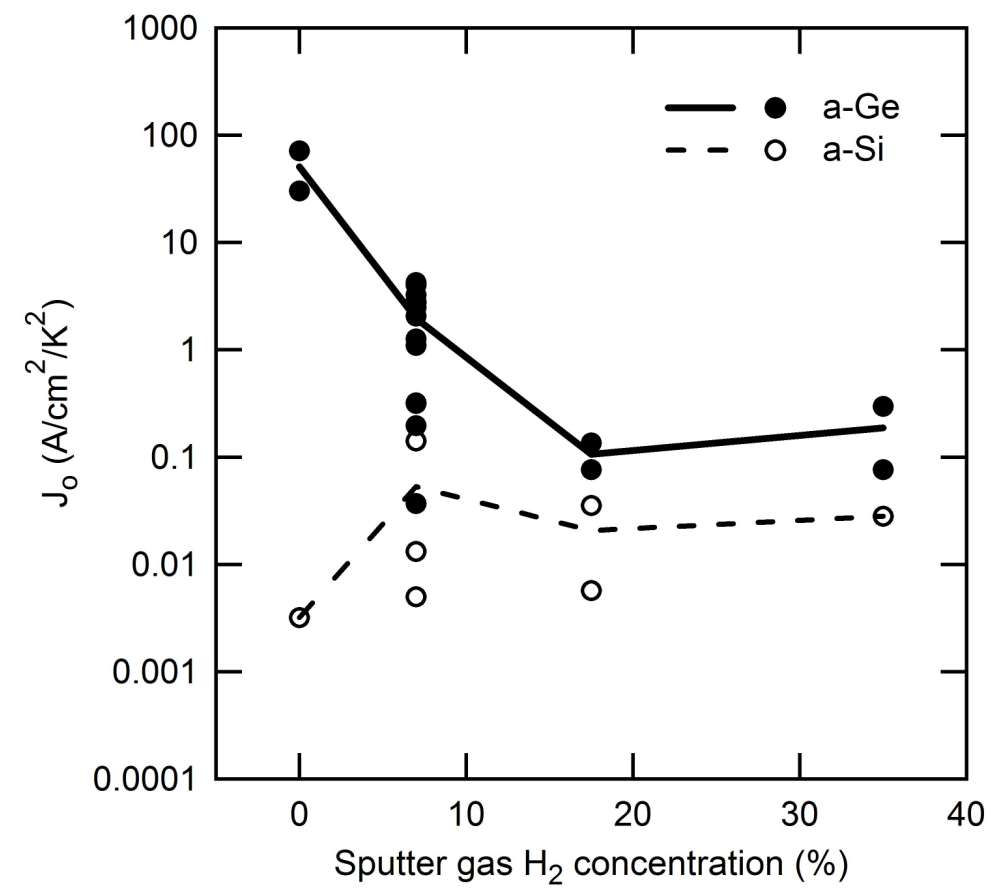

Figure 11. Electron injection current pre-factor plotted as a function of sputter gas hydrogen concentration for a-Ge/HPGe/a-Ge and a-Ge/HPGe/a-Si detectors of the configuration shown in Figure 1. The pre-factor is that of the bottom contact. The a-Ge bottom contacts were sputtered at a pressure of 15 mTorr, and the a$\mathrm{Si}$ bottom contacts were sputtered at a pressure of $7 \mathrm{mTorr}$. The data points include detectors produced from ORTEC1, ORTEC2, and LBNL1 HPGe materials. 


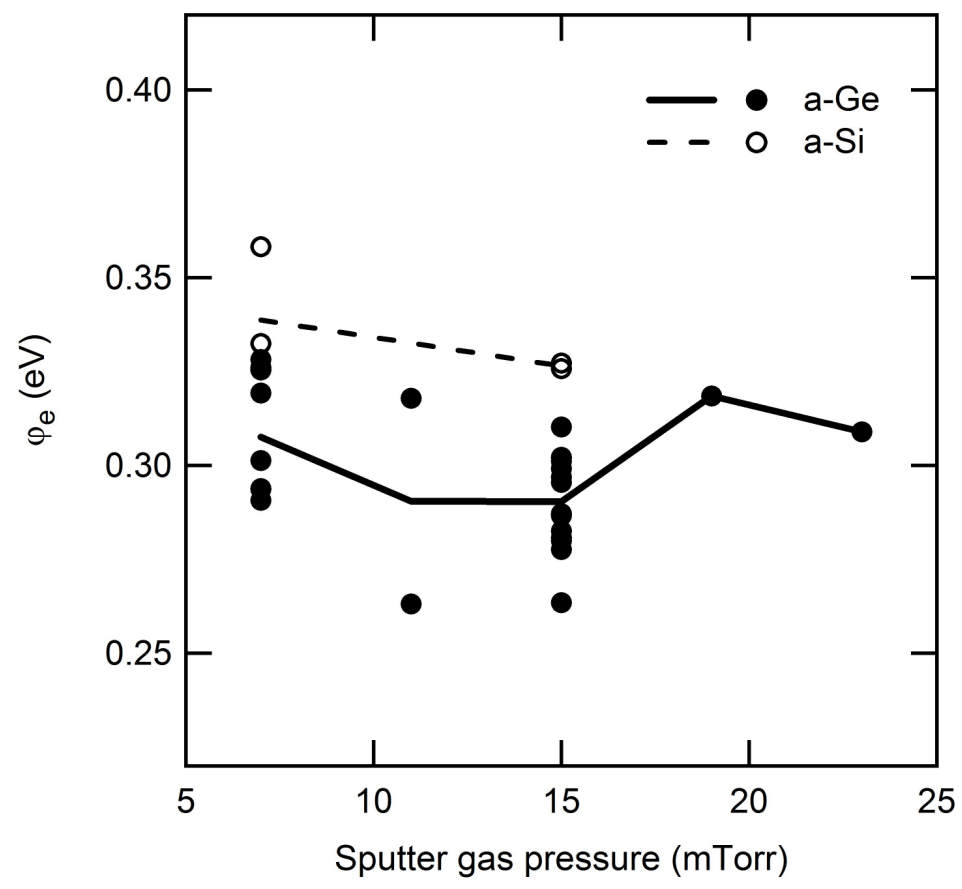

Figure 12. Electron energy barrier height plotted as a function of sputter gas pressure for a-Ge/HPGe/a-Ge and a-Ge/HPGe/a-Si detectors of the configuration shown in Figure 1. The barrier height is that of the bottom contact. The electrical contacts on the detectors were sputtered in Ar with $7 \% \mathrm{H}_{2}$. The data points include detectors produced from ORTEC1, ORTEC2, and LBNL1 HPGe materials. 


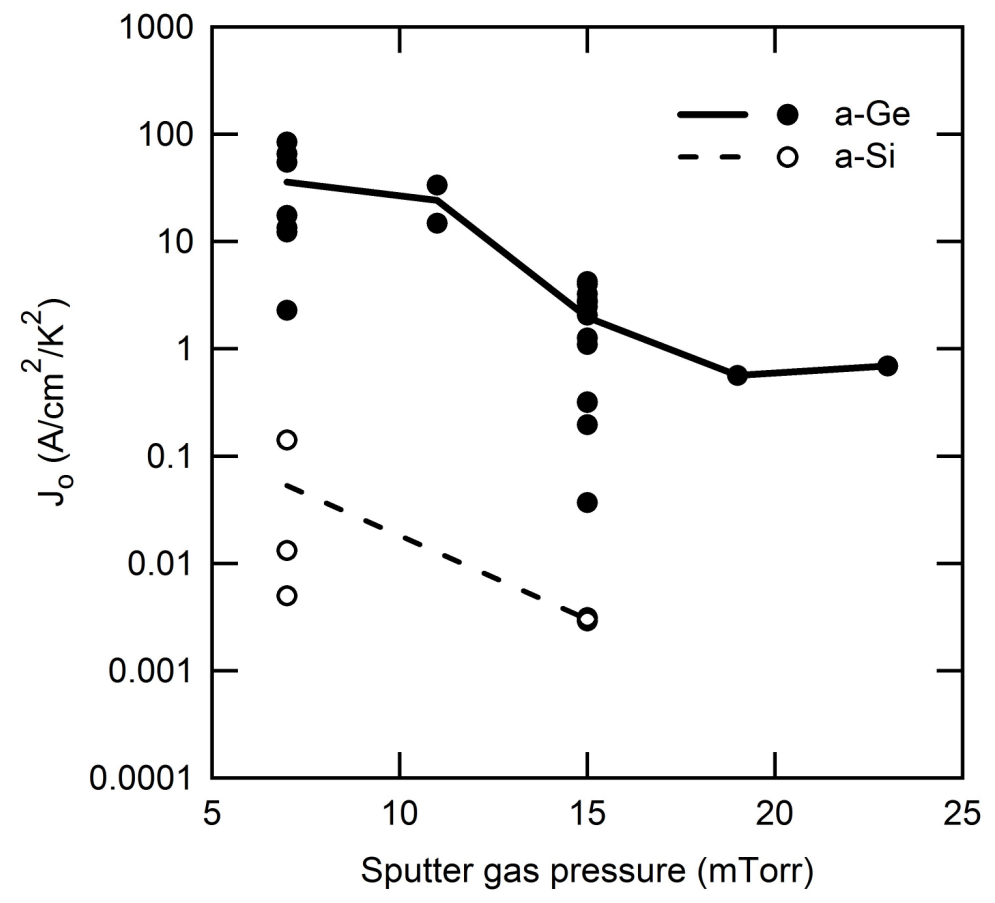

Figure 13. Electron injection current pre-factor plotted as a function of sputter gas pressure for a$\mathrm{Ge} / \mathrm{HPGe} / \mathrm{a}-\mathrm{Ge}$ and a-Ge/HPGe/a-Si detectors of the configuration shown in Figure 1. The pre-factor is that of the bottom contact. The electrical contacts on the detectors were sputtered in Ar with $7 \% \mathrm{H}_{2}$. The data points include detectors produced from ORTEC1, ORTEC2, and LBNL1 HPGe materials. 